Ann. Zootech., I980, 29 (2), I.37-I45.

\title{
Digestion dans l'intestin grêle chez le porc 1. - Définition des conditions d'obtention des digesta
}

\author{
Béattice DARCY et J. P. LAPLACE \\ Laboratoive de Physiologie de la Nutrition \\ Centre national de Recherches zootechniques, I.N.R.A., \\ $7835^{\circ}$ Jouy en Josas (France)
}

\begin{abstract}
Résumé
L'existence d'importantes perturbations du transit digestif lors de fistulation réentrante de l'iléon chez le Porc, et le problème soulevé par l'existence probable d'une fonction de nature sphinctérienne de la jonction iléo-caeco-colique dans cette espèce, sont rappelés. Les diverses techniques proposées font l'objet d'une analyse détaillée quant à la technologie des canules, à la technique chirurgicale, aux modalités d'implantation (iléo-iléale ou iléo-caecale) et à l'entretien des préparations.

L'évaluatıon critique de ces divers éléments bibliographiques dans la perspective d'une étude de bilan de la digestion dans l'intestin grêle du Porc, conduit à énoncer les principes essentiels qui doivent être respectés pour que les digesta collectés soient effectivement tels qu'ils sont normalement émis vers le gros intestin. Cette définition des conditions d'obtention des digesta amène à concevoir une technique chirurgicale nouvelle dénommée " fistulation iléo-colique postvalvulaire " dont la validation fera l'objet de travaux ultérieurs.
\end{abstract}

\section{Introduction}

Par rapport à la mesure de la digestibilité apparente globale des aliments, telle qu'elle peut être réalisée au niveau des fèces, l'obtention de digesta en divers points du tractus digestif autorise la détermination d'une digestibilité partielle. On peut ainsi, par exemple, évaluer l'utilisation digestive apparente des aliments au niveau gastro-duodénal ou au niveau de la jonction iléo-caeco-colique. Dans son principe cette méthode repose donc sur l'usage d'une fistulation chronique $\mathrm{du}$ tube digestif et sur la collecte quantitative des digesta. La même méthodologie permet l'étude cinétique des modifications qualitatives subies par les digesta en amont du site de leur dérivation.

De fait, les techniques de fistulation, simple ou réentrante, sont largement utilisées à tous les niveaux du tube digestif dans la quasi totalité des espèces 
animales domestiques. Ia fistulation réentrante, applicable en tout point de l'intestin grêle selon les principes généraux établis par Markowitz, ArchibaLD et Downik (1964), offre des avantages spécifiques pour une étude cinétique des transformations quantitatives et qualitatives des matériaux alimentaires au cours de leur digestion. La fistulation réentrante de l'iléon terminal, qui autorise un bilan de la digestion à la fin de l'intestin grêle est couramment utilisée sans difficultés majeures chez les ruminants (AsH, I962; Coombe et KAy, I965; MAC RaE et Armstrong, I969; Mac RaE, I974).

Par contre, dans le cas du Porc, sa mise en œuvre entraîne d'importantes difficultés que ne surmonte entièrement aucune des variantes techniques ou conditions d'utilisation jusqu'ici proposées dans la littérature. Dans un précédent travail (LAPLACE et BORGIDA, I976) il a été tenté d'identifier les causes des désordres rencontrés. De fait, la jonction iléo-caeco-colique chez le Porc semble présenter les caractères fonctionnels d'un véritable sphincter, comme chez l'Homme ou les Carnivores. Cette observation est étayée par la description histologique du renforcement considérable des couches musculaires circulaires au niveau de la jonction et notamment de la saillie iléale dans la lumière caeco-colique (BLESA SANCHEZ, I978). Aussi, tant en raison du mauvais fonctionnement habituel des fistules iléales réentrantes chez le porc qu'en raison du rôle physiologique potentiel de ce pseudo-sphincter iléo-caecal, il paraît essentiel de rechercher des conditions correctes d'obtention des digesta en vue d'études du bilan de la digestion à la fin de l'intestin grêle.

Dans cette perspective le présent travail se propose, au travers d'une analyse des techniques existantes, de procéder à l'évaluation critique de leurs avantages et inconvénients afin de dégager les principes requis pour l'obtention des digesta à la fin de l'intestin grêle dans de bonnes conditions.

\section{Les techniques existantes}

L'analyse des solutions à ce jour proposées doit prendre en considération les aspects suivants : technologie des canules, technique chirurgicale pour leur mise en place dans la lumière digestive, modalité de l'implantation réentrante, soins et maintenance post-opératoires, et méthodologie de collecte.

\section{I. - Technologie des canules}

Les canules utilisées sont le plus souvent en matériaux plastiques, tels que polyéthylène (CUNNINGHAM, Frifind et Nicholson, I962; EASTER et TANKSLEy, I973), ou chlorure de polyvinyle (HorszczAruK et ZEBrowskA, I973; IVAN, I974; BRAUde, Fulford et LOW, I976; Drochner et HAzeM, I976). La médiocre tolérance tissulaire de ces matériaux chez le Porc peut être améliorée par habillage de la canule à l'aide d'un film de silicone (EASTER et TANKSLEY, I973). Des canules réalisées entièrement en silicone médical allient plasticité et innocuité tissulaire (LAPLACE et TOMASSONE, I970; LAPLACE et BORGIDA, I976). Plus récemment ont été proposées des canules en verre pyrex, matériau rigide mais offrant à la fois une certaine tolérance tissulaire et une résistance modérée au glissement des contenus sur les parois (Livingstone et al., I977). 
En dépit de la variété des matériaux utilisés, la forme des canules est dans la majorité des cas identique ou analogue à celle d'une canule en T dont la partie endoluminale est constituée soit par une gouttière dans l'axe longitudinal du viscère, soit par une embase elliptique. Les canules en silicone de LAPLACE et TOMASSONE (I970) ne comportent par contre qu'un mince bourrelet endoluminal, la canule étant mise en place non plus perpendiculairement à l'axe du viscère, mais directement dans l'about intestinal sectionné. Enfin les canules proposées par IVAN (I974) sont particulièrement originales en ce qu'elles comportent un tube cylindrique endoluminal complet assurant le passage des digesta sans dérivation externe dans l'intervalle des collectes.

D'une façon générale, le calibre des tubes par lesquels passent les digesta est choisi aussi important que possible sous réserve de deux contraintes : la taille de l'animal donc le diamètre intestinal, et le calibre des tubes disponibles dans le commerce lorsque la canule n'est pas moulée à la demande. Pour des porcs d'un poids vif compris entre I4 et $30 \mathrm{~kg}$, DrochNER et HAZEM (I976) utilisent un tube de $12 \mathrm{~mm}$ de diamètre intérieur. Pour des porcs opérés à $30-35 \mathrm{~kg}$, que leur utilisation soit immédiate ou différée vers '50 kg, les diamètres intérieurs varient de i2 à $15 \mathrm{~mm}$ (Cunningham, Friénd et Nicholson, Ig62; Holmes, Bayley et Horney, I973; IVAN, I974; Braude, Fulford et Low, 1976). Sur des porcs de 45 à $55 \mathrm{~kg}$, EASTER et TANKSLEY (1973) emploient un tube de I2,8 mm de diamètre intérieur. De même, du tube de silicone de $\mathrm{I} 2,7 \mathrm{~mm}$ de diamètre intérieur a été utilisé chez des porcs de 55 à $75 \mathrm{~kg}$ (LAPLACE et BoRgIDA, I976). Les canules de verre de Lrvingstone et al., (r977) d'un diamètre intérieur de $8 \mathrm{~mm}$, seraient utilisables pour des porcs de 30 à $175 \mathrm{~kg}$.

\section{2. - Technique chirurgicale}

Dans la quasi totalité des cas, la mise en place des canules dans la lumière iléale répond à la technique initialement décrite et illustrée par MARKowiTz, ARchibald et Downie (I964). Les canules sont implantées respectivement dans les extrémités orale et aborale de l'intestin après transsection de celui-ci. Sur ce principe de base, une canule en 'T' est mise en place à la faveur d'une incision longitudinale sur le bord anti-mésentérique du moignon intestinal préalablement refermé. Deux variantes ont été utilisées, toujours après transsection de l'intestin : a) montage d'une canule en 'T dans l'extrémité du segment intestinal, obliquement par rapport à l'axe du viscère grâce à une découpe et une suture appropriées de l'about intestinal (HorszczaruK et zEBRowskA, I973); b) montage terminal selon l'axe du viscère d'un tube dépourvu d'embase endoluminale (LAPLACE et BORGIDA, I976).

Par rapport à ces techniques, celle de IVAN (I974) est particulièrement originale. Flle ne nécessite pas la transsection de 1'intestin, la dérivation externe des digesta n'étant qu'intermittente et obtenue par un dipositif spécial inhérent à la canule. Le tube cylindrique endoluminal est mis en place par incision longitudinale au bord anti-mésentérique.

\section{3. - Modalités de l'implantation réentrante}

Les principes ci-dessus concernent la mise en place des canules proximale et distale dans les abouts oral et aboral de l'iléon. Cependant pour éviter les fréquentes obstructions lors de ces fistulations réentrantes ileo-ileales, il a été proposé 
de réaliser une fistulation réentrante ileo-caecale (FASTER et TANKSLEY, I973; HAZEM et DrochNer, I976). Dans ce cas, la canule proximale est implantée comme indiqué ci-dessus, et la canule distale est une canule caecale conventionnelle. Ce principe n'exclut nullement la transsection iléale. Le moignon distal, qui ne porte pas de canule dans ce montage ileo-caecal, reste en place comme une courte anse aveugle auto-évacuante.

Quelle que soit la solution retenue, ileo-iléale ou ileo-caecale, les digesta sont donc toujours dérivés à une certaine distance en amont de la jonction ileo-caecocolique. $\mathrm{I}_{1}$ a localisation précise n'est pas systématiquement mentionnée par les auteurs, ou est repérée soit par rapport à la jonction iléo-caeco-colique, soit par rapport au ligament ileo-caecal. Néanmoins, dans l'ensemble, la dérivation des digesta intervient à environ $\mathrm{I} 3$ à $25 \mathrm{~cm}$ en amont de la jonction ileo-caeco-colique, indépendamment de la taille des animaux. Des dérivations plus proximales ont parfois été utilisées : I m (ZEBROWSKA, I973) voire I,5 m (LAPLACE et BORGIDA, I976) en amont de la jonction ileo-caeco-colique.

\section{4. - Entretien des préparations}

Selon les auteurs, le délai avant l'utilisation expérimentale de la préparation est essentiellement variable, de Io jours à I mois environ. En réalité, cette variabilité dépend surtout des modalités de la réalimentation post-opératoire. Divers auteurs recourent à une alimentation lactée exclusive durant 2 jours (EASTER et TANRSLEY, I973), 5 jours (HAZEM et DrochNer, I976), Io jours (DROChNeR et HAZEM, I976), voire 28 jours (Cunningham, Friend et Nrcholson, I962). Dans d'autres cas sont utilisés, en association avec le lait ou d'emblée, des régimes semi-synthétiques ou conventionnels, de mouture très fine (Horszczaruk et ZEBROWSKA, I973; IVAN, I974; BRAUdE, FllFord et LOW, I976). Un régime standard de mouture grossière n'a été utilisé qu'à titre de révélateur des difficultés de passage des digesta (LAPLACE et BoRGIDA, I976). Les thérapeutiques post-opératoires sont toujours analogues (antibiothérapie plus ou moins prolongée). L'utilisation des corticoïdes durant 7 jours n'a été proposée que par DROCHNER et HAZEM (I976). Enfin, les 2 canules sont toujours réunies entre elles par un tube extérieur à l'animal en dehors des collectes de digesta. Seule fait exception la préparation de Ivan (I974) excluant la dérivation externe dans 1'intervalle des expériences.

\section{Evaluation et critique méthodologique}

L'analyse des données réunies ci-dessus ne révèle pas au total de solution globalement adéquate. Au plan de la technologie des canules, l'usage du verre (LIVINGSTONE et al., I977) pourrait constituer un certain progrès, sinon quant aux propriétés physiques du matériau, du moins dans la mesure où les canules décrites par Livingstone et al. (I977) sont celles qui offrent le diamètre intérieur le plus important ( $8 \mathrm{~mm}$ ).

Les problèmes de forme des canules et de mise en place de celles-ci dans la lumière iléale ne peuvent être dissociés. La canule proposée par IvaN (I974) constitue un cas très particulier de canule en $T$, imposant à la fois la présence dans la lumière d'un tube rigide de $6,6 \mathrm{~cm}$ de long et son franchissement par les 
digesta sous le seul effet des pressions exercées en amont. La présence du tube dans la lumière iléale est susceptible d'une part de perturber voire empêcher le fonctionnement normal des reflexes entéro-entériques (FriEdman, I975), par non stimulation de la muqueuse sur une longueur de $6,6 \mathrm{~cm}$, d'autre part de susciter par son calibre important ( $9 \mathrm{~mm}$ de diamètre extérieur) un reflexe excitateur intestino-ileo-caecal dont l'existence parait probable chez le Porc (LAPLACE et BORGIDA, I976).

Par ailleurs, 1e franchissement d'un tube inerte par les digesta impose à l'intestin en amont de celui-ci un effort supplémentaire d'où résulte à brève échéance une hypertrophie de travail. Cet aspect n'a pas été examiné par IvaN (I974). Or une hypertrophie considérable a été constatée en amont d'un segment de tube de diamètre comparable, et d'une longueur de $10 \mathrm{~cm}$, interposé sur l'iléon après transsection à I $50 \mathrm{~cm}$ en amont de la jonction ileo-caeco-colique (LAPLACE et BORGIDA, I 976). Par ailleurs, ce tube est à lui seul capable d'entraîner l'obstruction irrémédiable en 3 jours de la lumière intestinale. Il est vrai que la canule d'IVAN (1974) n'implique pas une transsection comme c'est le cas pour l'interposition vraie d'un tube. Mais elle nécessite 4 ligatures circonférentielles enserrant l'intestin sur le tube rigide. Leur effet à l'égard de la propagation des phénomènes moteurs et de la conduction dans les plexus intra-pariétaux ne doit pas être sous estimé. Il semble donc que l'ingénieuse canule d'Ívan (I974) est malgré tout susceptible de provoquer des perturbations non négligeables même si l'auteur n'enregistre pas d'obstructions sous certaines conditions.

Les diverses canules en ' $\mathrm{T}$ ' conventionnelles, mises en place dans un about iléal, perpendiculairement, obliquement ou axialement, ne peuvent être classées objectivement. Tout au plus la réduction au minimum de la partie endoluminale de la canule et 1'obtention d'un passage direct bénéficient d'un a priori favorable En réalité, la question importante pour l'usage de ces canules est celle des modalités de l'implantation réentrante : ileo-iléale ou ileo-caecale.

Une préparation ileo-ileale conduit à dériver les digesta à une certaine distance en amont de la jonction ileo-caeco-colique. Ceci signifie que l'évaluation des caractéristiques des digesta parvenant au gros intestin est entachée d'erreur pour 3 raisons : a) il semble bien que la jonction iléo-caeco-colique chez le Porc constitue un véritable sphincter contrôlé par divers réflexes à partir de l'intestin grêle comme du gros intestin (LAPLACE et BorGIDA, I976) et qu'elle détermine donc la cinétique de passage d'un compartiment vers l'autre; b) l'iléon terminal paraît être le siège habituel d'une certaine accumulation de đigesta (LAPLACE, I975), or la présence en ce site d'une canule de dérivation autorise l'écoulement direct des digesta arrivant à ce niveau; c) la réintroduction des contenus s'effectue immédiatement en aval de la transsection intestinale i.e. dans le bref segment d'iléon terminal dont la motricité se trouve perturbée du fait de la transsection; ce phénomène s'ajoute à l'éventuelle difficulté de franchissement d'une longue tubulure inerte ( 2 canules et leur pontage externe dans l'intervalle des expérimentations.

Une préparation ileo-caecale peut faire l'objet de critiques identiques sur les deux premiers points : exclusion fonctionnelle de la jonction ileo-caeco-colique et dérivation prématurée des digesta. La difficulté mécanique de passage des contenus est certes moindre, avec diminution de l'incidence des obstructions (EASTER et 'TRANKSLEY, I973; HAZEM et DROCHNER, I976), mais une critique supplémentaire peut être formulée. L'accès direct à l'iléon offert à la flore caecocolique conduit à mettre en doute la validité des études de digestion fondées sur l'analyse de digesta collectés selon ce principe. Cette suspicion a priori ne 
pourra être confirmée ou infirmée que sur la base d'études qualitatives comparées des digesta obtenus selon les diverses techniques.

Enfin, aucune des précautions usuelles dans l'entretien post-opératoire des animaux ne résout les problèmes mécaniques rencontrés puisque la distribution d'une alimentation solide conduit toujours à des obstructions intempestives, même après une période d'alimentation lactée exclusive. L'usage permanent de régimes semi-synthétiques ou à mouture très fine ne constitue pas non plus une solution infaillible, confirmant par là le fait que les désordres observés sont dus à la préparation chirurgicale elle-même.

\section{Principes pour l'obtention des digesta au terme de la digestion dans l'intestin grêle}

De l'analyse critique qui précède, il ressort à l'évidence qu'aucune technique n'est entièrement satisfaisante. Néanmoins, il est possible de dégager un certain nombre de principes dont le respect devrait théoriquement permettre de collecter les digesta dans de bonnes conditions c'est-à-dire, dans la perspective d'un bilan de la digestion dans l'intestin grêle, tels qu'ils sont normalement émis vers le gros intestin. Ces quelques principes peuvent être énumérés comme suit :

I - Eviter toute transsection de 1'iléon distal;

2 - Exclure toute dérivation prématurée des digesta;

3 - Préserver le rôle fonctionnel de barrière mécanique et bactériologique du sphincter iléo-caeco-colique;

4 - Utiliser des canules d'un diamètre intérieur de l'ordre de $20 \mathrm{~mm}$;

5 - Limiter au strict minimum la longueur de tube inerte à franchir.

La mise en œuvre de ces principes apparaît très contraignante. Sont exclues (I) toutes les techniques classiques de fistulation réentrante iléo-iléale ou iléocaecale, précédemment analysées. Seule la technique d'IVAN (I974) évite toute transsection iléale. Elle conduit cependant à une dérivation prématurée des digesta et se trouve donc exclue également (2). En définitive, pour satisfaire simultanément aux trois premiers principes, il s'avère nécessaire de collecter les digesta en abordant par la lumière caeco-colique la saillie iléale à la jonction iléo-caeco-colique. L'exigence (4) de canules d'un diamètre important est relativement la plus aisée à résoudre, et reste impérative en particulier lors d'ingestion de régimes naturels à forte teneur en composants indigestibles par voie enzymatique dans 1'intestin grêle. Enfin, la limitation (5) de la longueur de tubulure que doivent franchir les digesta semble nécessaire pour la même raison que l'usage de canules larges. Satisfaire à ce principe pourrait conduire à exclure tout dispositif réentrant, c'est-à-dire à nécessiter une intervention mécanique ou humaine pour la restitution des digesta même dans l'intervalle des expérimentations.

Ces éléments conduisent donc à poursuivre le développement d'une technique de fistulation originale sur la base des premiers essais de montage en stomie cutanée d'un disque de paroi caeco-colique portant la saillie iléale terminale (LAPIACE et BorgIDA, I976). L'obtention d'un montage (fig. I) permettant à cette saillie iléale d'éjecter les digesta non plus dans la lumière caeco-colique, mais directement dans une canule de collecte permettrait de répondre à l'ensemble des principes définis plus haut. La restitution des digesta pourrait alors être assurée, par tout moyen à définir, à la faveur d'une canule mise en place dans le côlon proximal. 
Un tel montage pourrait être désigné sous le nom de fistulation iléo-colique postvalvulaive, en conservant le qualificatif usuel de valvule (ou valve) pour désigner la jonction iléo-caeco-colique.

Les premieres observations réalisées au terme d'une étude préliminaire de mise au point (DARCY, LAPLACE et VILLIERS, I980) suggèrent que l'usage de la fistulation ileo-colique post-valvulaire peut être valablement envisagé pour l'étude de la digestion dans l'intestin grêle du Porc. Sa validité, et les problèmes nouveaux qu'elle est susceptible de faire surgir, ne pourront être examinés que sur la base

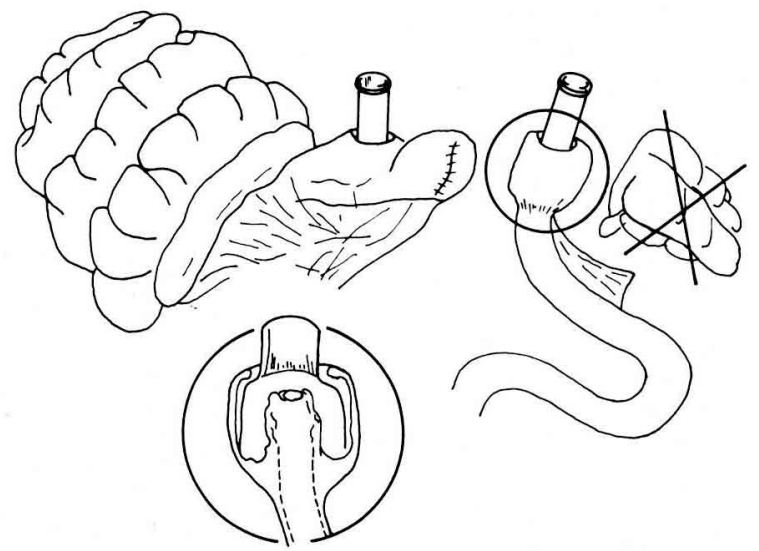

FIG. I. - Principe du montage dit "Fistulation iléo-colique post-valvulaive "

Cette technique consiste, après résection de la partie aveugle du caecum et transsection du côlon proximal, en la construction d'une petite poche de tissu caeco-colique (encerclée), montée sur une canule, et dans laquelle débouche l'iléon terminal (voir coupe en cartouche). Les digesta peuvent ainsi être recueillis tels qu'ils sont nomalement émis vers le gros intestin. Leur réintroduction est autorisée par une seconde canule mise en place dans l'about proximal refermé đu côlon hélicoïdal (à gauche).

Principle of the so-called "Post-valvular ileocolic fistulation "

This technique consists in a resection of the blind end of the caecum and a transsection of the proximal colon followed by the preparation of a small pouch of caecocolic tissue (encircled), fitted on a cannula, into which emerges the terminal ileum (see section in the separate circle). The digesta collected in this way correspond to those normally enitted into the large intestine. They are reintroduced into the intestine through a second cannula placed in the closed proximal end of the helicoidal colon (left).

d'études comparatives ultérieures. Parallèlement, une telle technique devrait permettre l'exploration de la fonction de la jonction iléo-caeco-colique chez le Porc.

Accepté pour publication en mars I980.

\section{Summary}

Digestion in the pig small intestine

I) Definition of digesta collection conditions

The existence of large disturbances of the gastro-intestinal food passage after re-entrant fistulation of the ileum in the pig, and the possible existence of a sphincter like function of the ileo-caeco-colic junction in this species, are recalled. The various techniques used are analysed, 
i.e. cannula technology, surgical technique, ways of re-entrant fistulation (ileo-ileal or ileo-caecal) and maintenance of preparation.

A critical examination of the information gathered from the literature intended for studying digestion balance in the small intestine leads to pointing out 5 essential principles which have to be respected in order that digesta collected may be like those normally emitted towards the large intestine. These principles are the following : (I) Avoid any transsection of the distal ileum; (2) Exclude a too early derivation of digesta; (3) Preserve the functional role of mechanical and bacterial barrier of the ileo-caeco-colic sphincter; (4) Use cannulas with an inside diameter of $20 \mathrm{~mm}$; (5) Reduce to the very minimum the length of passage through the inert tube.

These conditions used to obtain digesta require development of a new surgical technique called "post-valvular ileocolic fistulation", the principle of which is shown in figure I. The validity of this technique will be examined in further studies.

\section{Références bibliographiques}

Ash R. W., r962. Gastro-intestinal reentrant cannulae for studies of digestion in sheep. Anim. Prod., 4, 309-312.

BLESA SANChEZ E., 1978. Influencia de la reseccion de la union ileocecal en la absorcion de nutrientes en el cerdo. Thèse Doct. Med., Grenade, Avril 1978.

Braude R., FUlford R. J., I.W A. G., I976. Studies on digestion and absorption in the intestines of growing pigs. Measurements of the flow of digesta and pH. Br. J. Nutr., 36, 497-5 го.

COOMBE, J. B., KAY R. N. B., I965. Passage of digesta through the intestines of the sheep. Retention times in the small and large intestines. Br. J. Nutr., 19, 325-338.

Cunningham H. M., Friend D. W., Nicholson J. W. G., ig6z. Note on a reentrant fistula for digestion studies with pigs. Can. J. Anim. Sci., 42, I I -I I3.

DARCY B., LAPLACE J. P., VILLIERS P. A., I980. Obtention des digesta parvenant au gros intestin par fistulation ileo-colique post-valvulaire : Note préliminaire. Reprod. Nutr. Develop., 20, I $197-1202$.

DrochNeR W., HAZEM A. S. Von, 1976. Entwicklung einer Umleitungskanülentechnik für das Ileum beim Schwein. Z. Tierphysiol. Tierennähr. Futtermittelkde, 37, 26-3o.

EASTER R. A., TANKSLEY T. D., I973. A technique for reentrant ileo-caecal cannulation of swine. J. Anim. Soi. 36, I099-I I03.

Friedman M. H. F., I975. The entero-enteric reflexes, p. 57-73, in Friedman M.H.F., Functions of the stomach and intestine, I vol., University Park Press, Baltimore.

HAZEM A. S. Von, DrochnER W., I976. Extracorporaler ileocaecaler Bypass unter Umgehung des Ostium ileocaecale beim Schwein. Z. Versuchstierk., 18, 303-3o6.

HOLMES J. H. G., BAYLEY H. S., HORNEY F. D., 1973. Digestion and absorption of dry and high moisture maize diets in the small and large intestine of the pig. Br. J. Nutr., 30, 40I-4IO.

HorszczarUK F., ZEBRowSKA I., I973. Permanent intestinal fistulae for the study of digestion in pigs. 3) Establishment of reentrant fistulae of the small intestine (Polish). Roczn. Nauk. Roln. 95, I 57 -I 68 .

IVAN M., I974. A new type of reentrant cannula designed for use in the small intestine of the pig. Austr. Vet. J., 40, 547-552.

LAPLACE J. P., 1975. Le transit digestif chez les Monogastriques. II Phénomènes moteurs et mouvements des digesta. Ann. Zootech, 24, 489-55I.

LAPLACF, J. P., BORGid A L. P., I976. Problèmes physiologiques posés par la fistulation réentrante chronique de l'iléon chez le Porc. Étude bibliographique et expérimentale. Ann. Zootech., 25, $36 \mathrm{I}-37 \mathrm{I}$.

Laplace J. P., Tomassone R., I970. Evacuation gastro-duodénale chez le Porc. Fistulation chronique par voie thoracique extra-pleurale; recherche d'une technique d'analyse mathématique de l'évacuation. Ann. Zootech., 19, 303-332.

Iivingstone R. M., Fowlek V. R., White F., Wenham G., 1977. Annealed glass cannulae for use in digestion studies with pigs. Vet. Rec., 101, 368 . 
MaC RAE J. C., I974. The use of re-entrant cannulae to partition digestive function within the gastro-intestinal tract of ruminants, p. 26I-276, in Mc DONALD I. W., WARNER A. C. I., Digestion and Metabolism in the ruminant, University New-England Publication Unit, Armidale, Australia.

Mac RaE J. C., Armstrong D. G., i969. Studies on intestinal digestion in the sheep r) The use of chromic oxide as an indigestible marker, $B r . J$. Nutr., 23, I 5-23.

Markowitz J., ARchibaLD J., Downie H. G., I964. Experimental surgery. IX Intestinal fistulae : jejunostomy, p. I43-I63, 5th Ed., Williams Wilkins Co, Baltimore.

ZEBROWSKA T., I973. Influence of dietary protein source on the rate of digestion in the small intestine of pigs. I) Amount and composition of digesta. Roczn. Nauk. Roln., 95 B, I I5-I33. 